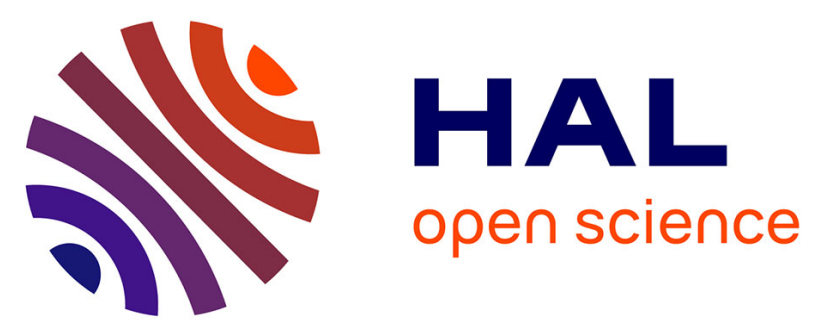

\title{
A photometric approach of fluorescent solar concentrators. Role of diffuse reflectors and spectral sensitivity of solar cells
}

J. Mugnier, Y. Dordet, Jean Pouget, B. Valeur

\section{- To cite this version:}

J. Mugnier, Y. Dordet, Jean Pouget, B. Valeur. A photometric approach of fluorescent solar concentrators. Role of diffuse reflectors and spectral sensitivity of solar cells. Revue de Physique Appliquée, 1987, 22 (1), pp.89-99. 10.1051/rphysap:0198700220108900 . jpa-00245520

HAL Id: jpa-00245520

https://hal.science/jpa-00245520

Submitted on 1 Jan 1987

HAL is a multi-disciplinary open access archive for the deposit and dissemination of scientific research documents, whether they are published or not. The documents may come from teaching and research institutions in France or abroad, or from public or private research centers.
L'archive ouverte pluridisciplinaire HAL, est destinée au dépôt et à la diffusion de documents scientifiques de niveau recherche, publiés ou non, émanant des établissements d'enseignement et de recherche français ou étrangers, des laboratoires publics ou privés. 


\title{
A photometric approach of fluorescent solar concentrators. Role of diffuse reflectors and spectral sensitivity of solar cells
}

\author{
J. Mugnier, Y. Dordet, J. Pouget and B. Valeur \\ Laboratoire de Chimie Générale $\left({ }^{*}\right)$, Conservatoire National des Arts et Métiers, 292, rue Saint-Martin, 75003 Paris, \\ France \\ (Reçu le 12 février 1986, révisé le 23 septembre, accepté le 26 septembre 1986)
}

\begin{abstract}
Résumé. - Une nouvelle méthode photométrique a été mise au point pour évaluer les performances des concentrateurs solaires fluorescents (CSF). Cette méthode permet de séparer la contribution relative du nombre de photons disponibles au niveau de la face de sortie des lames fluorescentes, et l'adaptation spectrale du flux lumineux à la sensibilité des photopiles. Deux lames ont été utilisées pour les expériences : une lame de polyméthacrylate de méthyle dopée avec un colorant organique (DCM) et une lame de rubis. Les résultats montrent que l'adaptation spectrale à la sensibilité des photopiles joue un faible rôle. Les améliorations de l'efficacité des CSF résultant de l'utilisation de réflecteurs diffusants sont discutées en relation avec l'effet de réabsorption et les défauts de la matrice.

Abstract. - A new photometric method has been developed for evaluation of the performances of fluorescent solar concentrators (FSC). This method allows to separate the relative contribution of the number of photons available at the output edge of the fluorescent plates, and the spectral matching of the radiant flux to the photovoltaic cell sensitivity. Experiments have been carried out on two plates : a plate of polymethylmethacrylate doped with an organic dye (DCM) and a ruby plate. The results show that the spectral matching to the cell sensitivity plays a minor role. The improvements in FSC efficiency thanks to diffuse reflectors are discussed in conjunction with the reabsorption effect and the defects of the matrix.
\end{abstract}

\section{Introduction.}

Fluorescent solar concentrators (FSC) have been the subject of significant attention over the last few years [1-7]. The principle of FSC operation is based on the absorption of solar radiation by a plate containing a fluorescent species whose emission band overlaps as little as possible the absorption band. The fluorescence photons are trapped within the plate and transported by total internal reflection to the edge of the plate where they are converted by a photovoltaic cell coupled to the plate with an optical fluid. Diffuse reflectors may be used to increase the efficiency of the system.

The main limitations of FSC are due to the fluorescent species and to the properties of the plate. With regard to the former,

- the absorption region is often narrow with respect to the solar spectral flux ;

(*) Unité associée au CNRS $\mathrm{n}^{\circ} 1103$, Physico-chimie Organique Appliquée.
- the fluorescence quantum yield is currently lower than 1 ;

- the spectral overlap of the absorption and emission bands is often large, thus leading to reabsorption phenomena ;

- the emission band is not always well suited to the spectral sensitivity of the cells ;

- the photochemical stability of organic fluorophores is often poor.

Limitations are also due to the plate :

- only a part of the fluorescence photons is trapped ;

- a lack of optical quality causes some photons to be lost by absorption and by scattering inside the plate or at the surface.

On the other hand, FSC offer distinct advantages in comparison with other types of concentrators :

- they collect both direct and diffuse light ;

- there is good heat dissipation of non utilized 
energy by the large area of the collector plate in contact with air ;

- tracking the sun is unnecessary.

Various fluorescent materials either organic or inorganic have been used and several matrices (liquid $[6,8]$, polymer [2-5, 9], glass [10-13]) have been proposed. Many investigations have been devoted to FSC [4, $5,7,14-17]$ in order to examine the reasons of their limited performances.

One aspect of FSC has drawn little attention so far [18]: it concerns the spectral matching of the flux (emitted by the plate) to the cell sensitivity. Thus, considering the output edge of the plate as a source whose spectral radiant emittance is known, it is of interest to separate the relative contribution of the number of collected photons available at the edge of the plate, and the spectral matching of this flux to the cell sensitivity.

Measurements of the spectral flux emitted by the plate will allow us to examine the effects of different reflectors used in our device.

Two types of plate will be examined. Firstly, a plate of PMMA (polymethylmethacrylate) doped with an organic dye, DCM (4-dicyano-methylene-2-methyl-6-pdimethylaminostyryl-4H-pyran). This dye has been often used in FSC because of its large absorption band, its good quantum yield and the poor spectral overlap of the absorption and emission bands [5]. Secondly, a ruby plate (chromium doped sapphire) which is unalterable and exhibits broad absorption bands, good quantum yield and emission at about $700 \mathrm{~nm}$, which is close to the maximum of sensitivity of silicon cells. Moreover the mechanical and optical properties of ruby are excellent. This material was previously suggested for solar applications [19]. Its properties and its remarkable performances in FSC [20] led us to propose it as a model material for FSC.

After describing the characteristics of FSC operation, and the effects of diffuse reflectors, we shall present a new method for examining the importance of the spectral sensitivity of the cell.

\section{Characteristics of FSC operation. Role of diffuse reflectors.}

The most evident feature of a FSC is its geometric gain $G$ which is the ratio of the collecting area $S$ to the output edge area $s_{\mathrm{e}}$

$$
G=\frac{S}{S_{\mathrm{e}}}
$$

The power gain is the ratio of the output power of the solar cell in the FSC to the output power of the same cell under direct insolation. Thus, we shall consider hereafter the incident flux on the FSC only in the range of spectral sensitivity (SS) of the cell. A silicon cell has been used in the present study.
Let $\phi_{\text {iss }}(\lambda)$ be the incident spectral radiant flux $\left(\mathrm{W} \cdot \mathrm{nm}^{-1}\right.$ ) normal to the collecting area $S$ of the FSC (Fig. 1). Part of this flux is reflected by the plate according to the reflection coefficient $r$ which depends on the refractive index $n$. It is to be noted that $n$ is wavelength dependent, but this dependence is weak in the spectral range under consideration and will be ignored. If no antireflection coating is used, the coefficient $r$ for unpolarized light is given by the Fresnel equation :

$$
r=\frac{(n-1)^{2}}{(n+1)^{2}}
$$

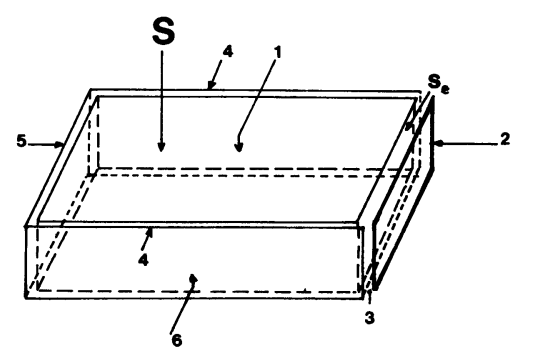

A)

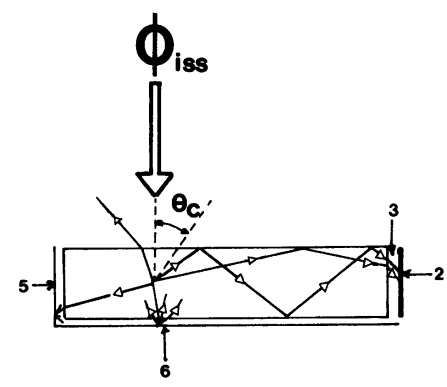

B)

Fig. 1. - FSC schematic. A. Parts of a FSC : 1, fluorescent plate ; 2, photovoltaic cell ; 3 , air gap ; 4, lateral diffuse reflector; 5, opposite diffuse reflector; 6 , back diffuse reflector $; S$, collecting area $; s_{e}$, output edge area. B. Principle of FSC operation (cross-section) $: \phi_{\text {iss }}$, incident radiant flux ; $\theta_{c}$, critical angle for internal reflection.

The transmittance $T(\lambda)$ of the plate is assumed to obey the Beer-Lambert relation :

$$
T(\lambda)=\mathrm{e}^{-\alpha(\lambda) d}
$$

where $\alpha(\lambda)$ is the absorption coefficient of the fluorophore and $d$ is the thickness of the plate.

In the device considered in this work (Fig. 1), a back diffuse reflector is coupled to the plate by an air gap. Assuming that this reflector is perfect, the total flux $\varphi_{\mathrm{a}}$ of photons absorbed by the plate within the spectral sensitivity (SS) of the solar cell is given by :

$$
\begin{aligned}
\varphi_{\mathrm{a}}=\int_{\mathrm{ss}} \phi_{\mathrm{iss}}(\lambda) & \cdot(1-r) \times \\
& \times\left[1-T(\lambda) \cdot T^{\prime}(\lambda)\right] \frac{\lambda}{h c} \mathrm{~d} \lambda
\end{aligned}
$$


where $h$ is Planck's constant, $c$ is the velocity of light and $T^{\prime}(\lambda)$ is the transmittance of the plate for the flux re-entering into the plate after reflection on the back reflector (see appendix).

Uppon the absorption of photons, the fraction of excited dye molecules that emit a fluorescence photon is equal to the quantum yield $\eta$. This quantum yield depends on the nature of the matrix and may also depend on the excitation wavelength.

The trapping yield $P_{\mathrm{c}}$ (fraction of trapped photons) is governed by the refractive index $n$ of the plate. The value of $n$ is related to the critical angle $\theta_{c}$ for total internal reflection :

$$
\theta_{\mathrm{c}}=\sin ^{-1}(1 / n) \text {. }
$$

The trapping yield is thus given by :

$$
P_{\mathrm{c}}=\cos \theta_{\mathrm{c}} \text {. }
$$

Let us consider now the fraction $P_{\mathrm{s}}$ of fluorescence photons escaping the plate through the output edge (section $s_{\mathrm{c}}$ ). In a first approach, we do not consider the reabsorption phenomenon and we use the following Keil's statement [21] : an air-gap coupled diffuse reflector can be regarded as a specular reflector of same reflectivity ( 1 for a perfect reflector) when used on a rectangular body.

The photovoltaic cell being coupled to the plate with an air gap, different expressions for $P_{\mathrm{s}}$ can be derived according to the number of diffuse reflectors (Fig. 1) :

- For a plate without reflector :

$$
P_{\mathrm{s}}=\frac{1-\cos \theta_{\mathrm{c}}}{2} \text {. }
$$

- For a plate with an opposite air-gap coupled reflector :

$$
P_{\mathrm{s}}=1-\cos \theta_{\mathrm{c}} .
$$

- For a plate with opposite and lateral air-gap coupled reflectors, Keil [21] has shown that the escape probability is the same as in the previous case. The lateral reflectors play a role only when the plate is coupled to the cell with a liquid for optical matching.

It should be emphasized that the contribution of a back reflector is only to increase the fraction of absorbed photons with almost no change in the escape probability [22].

In the ideal case of a perfectly polished and transparent plate doped with fluorophores whose absorption and emission bands do not overlap, the theoretical total flux of photons emitted by the plate is given by

$$
\begin{aligned}
\varphi_{\mathrm{e}}^{\mathrm{th}}=\int_{\mathrm{ss}} \phi_{\mathrm{iSS}}(\lambda) \cdot(1-r) & {\left[1-T(\lambda) \cdot T^{\prime}(\lambda)\right] \times } \\
& \times \eta(\lambda) \cdot P_{\mathrm{s}} \frac{\lambda}{h c} \mathrm{~d} \lambda .
\end{aligned}
$$

This expression takes into account the flux of absorbed photons, the quantum yield and the escape probability.

\footnotetext{
REVUE DE PHYSIQUE APPLIOUÉE. - T. 22, N 1, JANVIER 1987
}

Practically, a large part of the flux of emitted photons is lost by successive reabsorptions owing to the spectral overlap of absorption and emission. The self-absorption loss is a very difficult quantity to calculate exactly. Various theoretical and experimental studies have been devoted to this phenomenon [4, 7, 15-17]. Other attenuation effects are to be considered : absorption and scattering by the matrix imperfect internal multiple reflections due to surface defects [14].

\section{Photometric evaluation of performances.}

Goetzberger and coworkers [23] have proposed a model in which the optical efficiency of a FSC is assumed to be a product of independent terms characterizing the different loss mechanisms. In this global approach, the total incident flux is compared with the available total flux at the FSC output edge.

The aim of our approach is to compare the incident spectral flux to the emitted spectral flux with consideration of the spectral sensitivity of the cell. For this purpose, let $M(\lambda)\left(\mathrm{W} \cdot \mathrm{m}^{-2} \cdot \mathrm{nm}^{-1}\right)$ be the spectral radiant emittance of the output edge (area $s_{\mathrm{e}}$ ) of the FSC. The spectral radiant flux $\phi_{\mathrm{ed}}(\lambda)\left(\mathrm{W} . \mathrm{nm}^{-1}\right)$ is then:

$$
\phi_{\text {ed }}(\lambda)=\int_{0}^{s_{\mathrm{e}}} M(\lambda) \mathrm{d} s
$$

and the total emitted flux $\phi_{\text {ed }}(W)$ is given by :

$$
\phi_{\text {ed }}=\int_{\text {ss }} \phi_{\text {ed }}(\lambda) \mathrm{d} \lambda .
$$

If the spectral irradiance $E(\lambda)\left(\mathrm{W} \cdot \mathrm{m}^{-2} \cdot \mathrm{nm}^{-1}\right)$ on the plates is known, it is possible to calculate the number of photons that can reach the cell. The quantity of interest is the concentration in terms of number of photons $C_{n h \nu}$ which is the ratio of the number of photons collected by the edge-mounted cell, to the number of photons collected by the cell under direct insolation :

$$
C_{n h \nu}=\frac{\int_{0}^{s_{\mathrm{e}}} \int_{\mathrm{ss}} M(\lambda) \lambda \mathrm{d} \lambda \mathrm{d} s}{\int_{0}^{s_{\mathrm{e}}} \int_{\mathrm{ss}} E(\lambda) \lambda \mathrm{d} \lambda \mathrm{d} s} .
$$

Furthermore, the knowledge of the spectral sensitivity of the photovoltaic cell allows to evaluate the effect of the position and width of the fluorophore emission band. The spectral response of a cell reflects the probability that an electron-hole pair created by a photon at a given wavelength is collected in the junction. The energetic spectral response $R_{\mathrm{c}}\left(\mathrm{A} . \mathrm{W}^{-1}\right)$ represents the variation in short-circuit current density $J_{\mathrm{sc}}\left(\mathrm{A} \cdot \mathrm{m}^{-2}\right)$ as a function of the power density at each wavelength of the incident flux. It is then convenient to express the spectral effect of the fluorophores as the Stokes gain which is defined as : 


$$
G_{\mathrm{ST}}=\frac{I_{\mathrm{sc}}}{I_{\mathrm{sc}}^{0}}
$$

$I_{\mathrm{sc}}$ is the short-circuit current of the cell coupled to the plate, divided by the number of available photons, and $I_{\mathrm{sc}}^{0}$ is the short-circuit current of the same cell under direct insolation, divided by the number of incident photons in the region of spectral sensitivity of the cell. $I_{\mathrm{sc}}$ and $I_{\mathrm{sc}}^{0}$ are thus given by :

$$
\begin{aligned}
& I_{\mathrm{sc}}=\frac{\int_{0}^{s_{\mathrm{c}}} \int_{\mathrm{ss}} M(\lambda) \cdot R_{\mathrm{c}}(\lambda) \mathrm{d} \lambda \mathrm{d} s}{\int_{0}^{s_{\mathrm{c}}} \int_{\mathrm{ss}} M(\lambda) \cdot \frac{\lambda}{h c} \mathrm{~d} \lambda \mathrm{d} s} \\
& I_{\mathrm{sc}}^{0}=\frac{\int_{0}^{s_{\mathrm{e}}} \int_{\mathrm{ss}} E(\lambda) \cdot R_{\mathrm{c}}(\lambda) \mathrm{d} \lambda \mathrm{d} s}{\int_{0}^{s_{\mathrm{e}}} \int_{\mathrm{ss}} E(\lambda) \cdot \frac{\lambda}{h c} \mathrm{~d} \lambda \mathrm{d} s} .
\end{aligned}
$$

The concentration in terms of number of photons $C_{n h \nu}$ and the Stokes gain $G_{\mathrm{ST}}$ can be related to the gain in current intensity $G_{1}$ which is measurable with the cell alone. This gain is defined as the ratio of the shortcircuit current of the edge mounted cell to the value obtained with the same cell under direct insolation :

$$
G_{\mathrm{I}}=\frac{\int_{0}^{s_{\mathrm{e}}} \int_{\mathrm{ss}} M(\lambda) \cdot R_{\mathrm{c}}(\lambda) \mathrm{d} \lambda \mathrm{d} s}{\int_{0}^{s_{\mathrm{c}}} \int_{\mathrm{ss}} E(\lambda) \cdot R_{\mathrm{c}}(\lambda) \mathrm{d} \lambda \mathrm{d} s} .
$$

From the above definition of $C_{n h \nu}$ and $G_{\mathrm{ST}}$, we obtain :

$$
G_{\mathrm{I}}=G_{\mathrm{ST}} \cdot C_{n h \nu}
$$

This equation clearly shows that $G_{\mathrm{I}}$, which is easily measurable, is the product of two terms : one of them characterizes the spectral matching to the cell sensitivity and the other is related to the number of photons collected by the cell.

In addition, the quantum yield of the FSC can be defined as the ratio of the total flux of photons $\varphi_{\mathrm{e}}$ emitted through the output edge, to the total flux $\varphi_{\mathrm{a}}$ absorbed by the plate over the range of spectral sensitivity of the cell.

$$
\eta_{\mathrm{FSC}}=\frac{\varphi_{\mathrm{e}}}{\varphi_{\mathrm{a}}}
$$

This quantity is to be compared to $\eta_{\text {edge }}$ [9] which represents the ratio of the available power $(W)$ at the output edge, to the total incident power of the sun. However $\eta_{\text {FSC }}$ is relevant to numbers of photons instead of powers.

It is easy to show that $\eta_{\mathrm{FSC}}$ is given by :

$$
\eta_{\mathrm{FSC}}=\frac{C_{n h \nu}}{F_{\mathrm{a}} \cdot G}
$$

where $G$ is the geometric gain and $F_{\mathrm{a}}$ is the fraction of the incident flux of photons absorbed by the plate over the range of spectral sensitivity of the cell :

$$
F_{\mathrm{a}}=\frac{\int_{0}^{s} \int_{\mathrm{ss}} E(\lambda) \cdot(1-r)\left[1-T(\lambda) \cdot T^{\prime}(\lambda)\right] \lambda \mathrm{d} \lambda \mathrm{d} S}{\int_{0}^{s} \int_{\mathrm{ss}} E(\lambda) \lambda \mathrm{d} \lambda \mathrm{d} S} .
$$

\section{Experimental section.}

4.1 SOURCE. - A Luxarc 575 projector equipped with a $575 \mathrm{~W}$ Osram HMI metallogen lamp (colour temperature : $5600 \mathrm{~K}$ ) and a glass Fresnel lens were used for the simulation of the solar spectrum (close to AMO) without modification. The spectral radiant emittance of this source was compared with the emittance of a perfect black body, the temperature of which was measured by comparison with a standard lamp of known emittance at a given wavelength. In the 380$900 \mathrm{~nm}$ range, the irradiance was determined for a plane surface at a distance of $0.75 \mathrm{~m}$ from the source by using a calibrated Eppley thermopile equipped with a SFK 15 Schott filter. This calibration was performed at the Institut National de Métrologie (CNAM). Extension over the whole range of spectral sensitivity of the cell was possible thanks to Osram specifications of the lamp (Fig. 2). The total irradiance over this range (340$1120 \mathrm{~nm}$ ) is equal to $1430 \mathrm{~W} \cdot \mathrm{m}^{-2}$. Fluctuations in irradiance are less than $2 \%$ per hour. The homogeneity of irradiance was within $5 \%$ over an area of $105 \mathrm{~mm}$ of diameter, for a position of the Fresnel lens corresponding to an irradiance of $146 \pm 5 \mathrm{~W} \cdot \mathrm{m}^{-2}$.

Note that an accurate calibration of the source over the range of spectral sensitivity of the cell is required for the evaluation of FSC efficiency under actual conditions. Excitation energies below the gap of the cell should not be considered in this evaluation.

4.2 Plates. - One of the plates consists of PMMA doped with DCM (laser grade, from Exciton Chem. Co., used without further purification) at a concentration of $5.9 \times 10^{-4} \mathrm{~mol} . \mathrm{1}^{-1}$ (this value takes into 


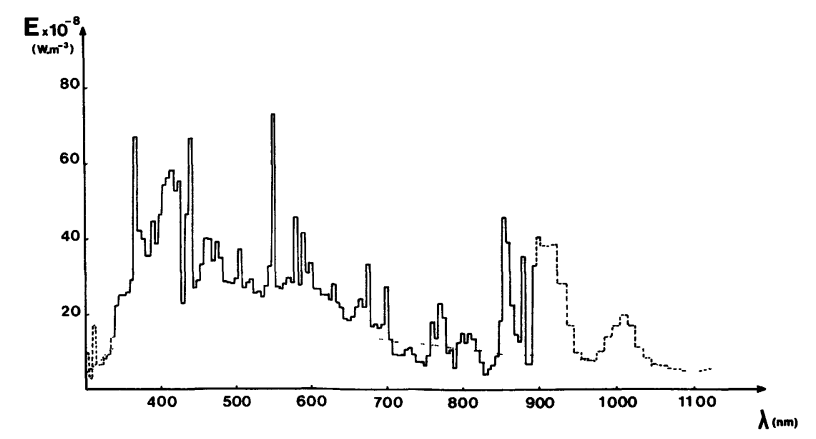

Fig. 2. - Comparison of the spectral irradiance of the HMI lamp (-) with the AMO solar spectral irradiance (.....). Extrapolation using OSRAM specifications is represented by a broken line.

account some skrinkage of PMMA during polymerization). This plate was made by CDF-chimie (oxygen-free process). The edges were polished with a sequence of grits, and the final buffing compounds were ALTUPOL 1 and 2.

The second plate is made of chromium doped sapphire (ruby). This material was suggested for the first time by Hovel and coworkers [19] for solar applications and especially when they are placed in optical series with the photovoltaic cells. Plates of ruby have been also proposed by us [20] as a model material for FSC. The properties of ruby are indeed very interesting. The mechanical properties are excellent (the hardness is 9 on the MOHS scale) which precludes any alteration of the surface after buffing. The refractive index of sapphire is close to 1.76 which ensures efficient trapping of light, and the transmittance is higher than $90 \%$ in the $250-5000 \mathrm{~nm}$ range. Ruby exhibits two broad absorption bands in the visible region. Its quantum yield is close to $1[19,24]$ and the spectral overlap of absorption and emission is very weak. Finally, ruby is an unalterable material and the emission spectrum is located around $700 \mathrm{~nm}$, i.e. in a region of high sensitivity of a silicon cell.

The weight-in concentration of chromium oxide in alumina was determined by atomic absorption and emission, and further confirmed by an optical method [25]. For the plate studied in this work, the concentration was found to be $(0.560 \pm 0.027) \%$. This plate with all buffed faces was purchased from Saphir France. It was oriented according to Buerger's method; the ternary axis is at $60^{\circ}$ with respect to the longest edge of the plate and almost in the plane of the collecting area S.

The dimensions of the plates were chosen according to different criteria : (i) A thickness of $1 \mathrm{~mm}$ is close to the optimal value reported in the literature [7] ; (ii) Previous works $[6,7]$ have shown that the geometry of the collecting area has slight effects on the FSC efficiency; (iii) In our experimental set-up, the homogeneity of the irradiance was satisfactory over an area of $105 \mathrm{~mm}$ of diameter ; (iii) The manufacture of large and thin ruby plates is difficult. All these considerations led us to choose rectangular plates : $100 \mathrm{~mm} \times$ $20 \mathrm{~mm} \times 1 \mathrm{~mm}$. Using the shortest edge $\left(s_{\mathrm{e}}=20 \mathrm{~mm}^{2}\right)$ as the output edge, the geometric gain $G$ is equal to 100.

4.3 RefleCtORS. - The diffuse reflectors (see Fig. 3) are made of Cegedur-Pechiney aluminum mirrors covered with six coats of paint (Eastman-Kodak White Reflectance Coating) whose reflection efficiency is close to 1 [26]. The ruby plate and the doped PMMA plate, equipped with back, lateral and opposite reflectors will be hereafter called device 1 and device 2, respectively.

4.4 Рнотovoltaic Cell. - The np type singlecrystal silicon cell SAT 302 (size : $20 \times 20 \mathrm{~mm}^{2}$; resistivity : $10 \Omega . \mathrm{cm}$ ) was obtained from the Société Anonyme des Télécommunications. Cleavage and

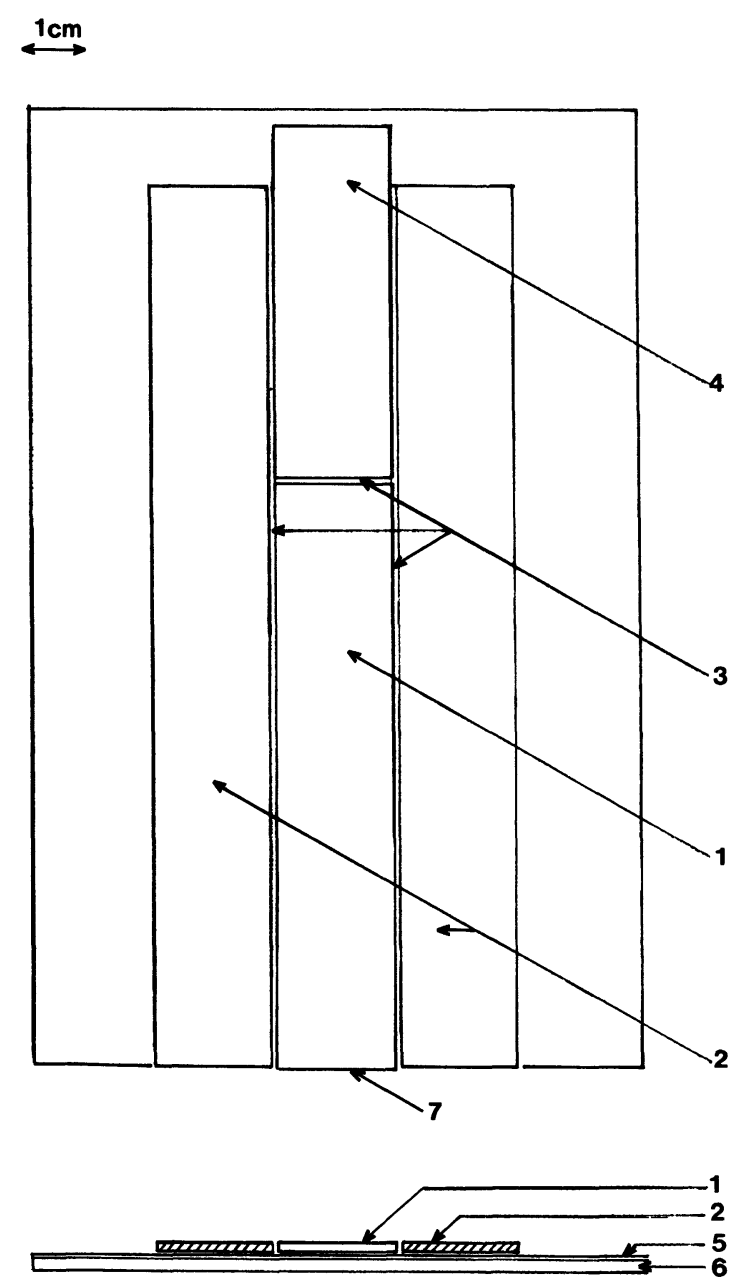

Fig. 3. - Diagram of the devices under study : 1, fluorescent plate ; 2 , movable lateral reflectors ; 3 , air gaps ; 4, movable opposite reflector; 5 , back reflector; 6 , holder ; 7 , output edge either coupled to the cell or penetrating the integrating cylinder. 
masking tests were carried out in order to select the best way to use the cell at the output edge of the FSC $\left(20 \times 1 \mathrm{~mm}^{2}\right)$. The best results were obtained by carefully masking the cell which led to a fill factor of 0.62 and to a series resistance of $5 \Omega$. Under the illumination conditions described in section 4.1 , the short-circuit current $I_{\mathrm{sc}}^{0}$ and the open-circuit voltage were found to be $1.49 \mathrm{~mA}$ and $398 \mathrm{mV}$, respectively. The relative spectral sensitivity $\left(A . \mathrm{W}^{-1}\right)$ of the cell was determined at intervals of $20 \mathrm{~nm}$. By using neutral filters, the incident energy was kept constant and the variation in short-circuit current as a function of incident flux was checked. The excitation beam from a $450 \mathrm{~W}$ xenon arc lamp was passed through a monochromator. By means of a movable mirror at the output of the monochromator, the excitation beams was periodically deflected towards a thermophile (Hilger \& Watt type F 10) whose sensitivity is constant over the 0.18-3.4 $\mu \mathrm{m}$ range. Modulation of the beam at $15 \mathrm{~Hz}$ and synchronous detection amplification ensure good accuracy and stability. The current was measured with a AOIP MNK 177 multimeter. The relative error in the determination of relative spectral sensitivity was about $3 \%$ (Fig. 4).

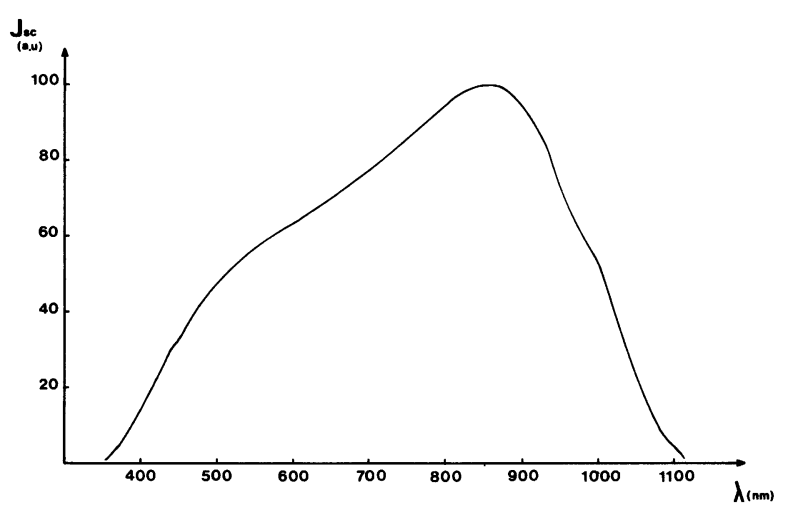

Fig. 4. - Relative spectral sensitivity of the cell (SAT 302).

4.5 THE « ICOFMP » SYSTEM. ABSORPTION AND FLUORESCENCE MEASUREMENTS. - The available flux at the output edge of the plate was measured with a home-made integrating cylinder (diameter: $70 \mathrm{~mm}$, height : $40 \mathrm{~mm}$ ). The interior was covered with six coats of paint (Eastman-Kodak White Reflectance Coating). The FSC plate penetrates the cylinder by $0.5 \mathrm{~mm}$ through a tunable slit along a generant. An optical multimode fiber (Fibre Optique Industrie ; diameter : $1 \mathrm{~mm}$ ) which is connected to the circular face of the cylinder through a coupling-pipe, transmits the fluorescence signal to the detection system (monochromator, photomultiplier, electronics) of an AMINCO SPF 500 spectrofluorometer interfaced to a Kontron PSI 80 microcomputer for storage, correction and data analysis. The whole system consisting of the integrating cylinder, the optical fiber, the monochromator and the photomultiplier (Hamamatsu R 777) will be called ICOFMP.

Since ruby is an anisotropic material, the absorption spectrum cannot be obtained on a conventional spectrophotometer. This spectrum was recorded by using the natural light of the HMI lamp and the ICOFMP system. In this case, the input slit of the cylinder was replaced by a circular hole of $3 \mathrm{~mm}$ of diameter. The doped PMMA plate was studied under the same conditions and the absorption spectrum was compared to that recorded on a Kontron UVIKON 820 spectrophotometer. A comparison of the spectra shows that the results are consistent within $1 \%$.

4.6 CAlibration OF THE ICOFMP System. FluX MEASUREMENTS. - Exitance at the output edge of the plates was shown to be constant over the whole section $s_{\mathrm{e}}$. The variations did not exceed $0.5 \%$, except at the extremities $(2 \%)$. These results were obtained by moving the integrating cylinder, the slit being replaced by a $0.25 \mathrm{~mm}$ hole (as in the absorption measurements on the ruby plate). Consequently, the flux measured by the ICOFMP system will be proportional to the collecting or emitting surfaces.

Calibration of the ICOFMP system was done under illumination of the slit of the integrating cylinder with the HMI lamp under standard conditions (see Sect. 4.1). The spectral flux $\phi_{\mathrm{m}}(\lambda)$ recorded by the ICOFMP system is related to the known irradiance $E(\lambda)$ according to the following equation :

$$
\phi_{\mathrm{m}}(\lambda)=E(\lambda) \cdot S_{\mathrm{c}} \cdot K(\lambda)
$$

where $S_{\mathrm{c}}$ is the surface of the slit (opened at the maximum for this measurement) and $K(\lambda)$ is the response of the ICOFMP system. $K(\lambda)$ was determined up to $850 \mathrm{~nm}$ (this wavelength corresponds to the limit of the sensitivity range of the photomultiplier). The spectral range of this calibration is satisfactory in regard to the emission spectra of the ruby and doped PMMA plates.

For each plate, the input slit of the cylinder fits exactly the section $s_{\mathrm{e}}$ of the plate. The measured flux is thus given by :

$$
\phi_{\mathrm{em}}(\lambda)=M(\lambda) \cdot s_{\mathrm{e}} \cdot K(\lambda) .
$$

Then, the expressions for the concentration in number of photons $C_{n h \nu}$ (Eq. (12)), the Stokes gain $G_{\mathrm{ST}}$ (Eq. (13)) and the gain in current intensity $G_{\mathrm{I}}$ (Eq. (16)) can be written in the simple following forms :

$$
C_{n h \nu}=\frac{\frac{S_{\mathrm{c}}}{s_{\mathrm{e}}} \int_{\mathrm{ss}} \frac{\phi_{\mathrm{em}}(\lambda)}{\phi_{\mathrm{m}}(\lambda)} E(\lambda) \cdot \lambda \mathrm{d} \lambda}{\int_{\mathrm{ss}} E(\lambda) \cdot \lambda \mathrm{d} \lambda}
$$




$$
\begin{gathered}
G_{\mathrm{ST}}=\frac{\int_{\mathrm{ss}} \frac{\phi_{\mathrm{em}}(\lambda)}{\phi_{\mathrm{m}}(\lambda)} E(\lambda) \cdot R_{\mathrm{c}}(\lambda) \cdot \mathrm{d} \lambda \cdot \int_{\mathrm{ss}} E(\lambda) \cdot \lambda \mathrm{d} \lambda}{\int_{\mathrm{ss}} \frac{\phi_{\mathrm{em}}(\lambda)}{\phi_{\mathrm{m}}(\lambda)} E(\lambda) \cdot \mathrm{d} \lambda \cdot \int_{\mathrm{ss}} E(\lambda) \cdot R_{\mathrm{c}}(\lambda) \cdot \mathrm{d} \lambda} \\
G_{\mathrm{I}}=\frac{\frac{S_{\mathrm{c}}}{s_{\mathrm{e}}} \int_{\mathrm{ss}} \frac{\phi_{\mathrm{em}}(\lambda)}{\phi_{\mathrm{m}}(\lambda)} E(\lambda) \cdot R_{\mathrm{c}}(\lambda) \cdot \mathrm{d} \lambda}{\int_{\mathrm{ss}} E(\lambda) \cdot R_{\mathrm{c}}(\lambda) \cdot \mathrm{d} \lambda} .
\end{gathered}
$$

It should be noted that, as expected, the Stokes gain (Eq. (24)) does not depend on the slit aperture. After determination of the fraction $F_{\mathrm{a}}$ of the incident flux absorbed by the plate (Eq. (20)), the quantum yield $\eta_{\text {FSC }}$ for a known geometric gain $G$ can be calculated by means of equation (19).

Furthermore, the knowledge of the absolute spectral irradiance $E(\lambda)$ allows to calculate the absolute values of $\phi_{\mathrm{em}}$ by means of equations (21) and (22).

\section{Results and discussion.}

The ICOFMP system has been used to determine the wavelength dependence of the flux emitted by the plates in devices 1 (ruby) and 2 (DCM in PMMA). The efficiency of the reflectors will be examined. Then it will be possible to determine $C_{n h \nu}$ and $G_{\mathrm{ST}}$ for both devices. The value of $G_{\mathrm{I}}$ that can be calculated from these results will be compared to the value directly obtained by using the air-gap coupled cell.

5.1 SPECTROSCOPIC CHARACTERISTICS OF THE PLATES. - For an optical path of $1 \mathrm{~mm}$, the absorption spectra of the two plates are reported in figure 5. Ruby exhibits two broad absorption bands $\left(\lambda_{\max }=405\right.$ and $565 \mathrm{~nm}$ ) like organic dyes. In addition to these bands, a weak absorption band ( $\mathrm{R}$ band) is located at $694 \mathrm{~nm}$; this band will be responsible for reabsorption effects in this material. For DCM, the wavelength of maximum absorption is $461 \mathrm{~nm}$.

The corrected emission spectra given in figure 5 are normalized to the same height at the maximum. The position of the emission maximum of a PMMA plate doped with DCM at low concentration $\left(10^{-6} \mathrm{~mol} \mathrm{l}^{-1}\right)$ is $550 \mathrm{~nm}$, whereas for the plate used in device 2 , the maximum is red shifted by $50 \mathrm{~nm}$ (under illumination by the HMI lamp). This shift is due to the reabsorption phenomenon. Furthermore, the emission spectrum of a ruby plate at low chromium concentration $\left(\mathrm{Cr}_{2} \mathrm{O}_{3} / \mathrm{Al}_{2} \mathrm{O}_{3} \sim 0,12 \mathrm{w} / \mathrm{w}\right)$ exhibits the characteristic bands $R_{1}(\lambda=694 \mathrm{~nm})$ and $R_{2}(\lambda=$ $693 \mathrm{~nm}$ ), whereas for the ruby plate used in device 1 , these bands are decreased owing to the reabsorption effect, and emission in the 700-750 $\mathrm{nm}$ region appears more distinctly after normalization.

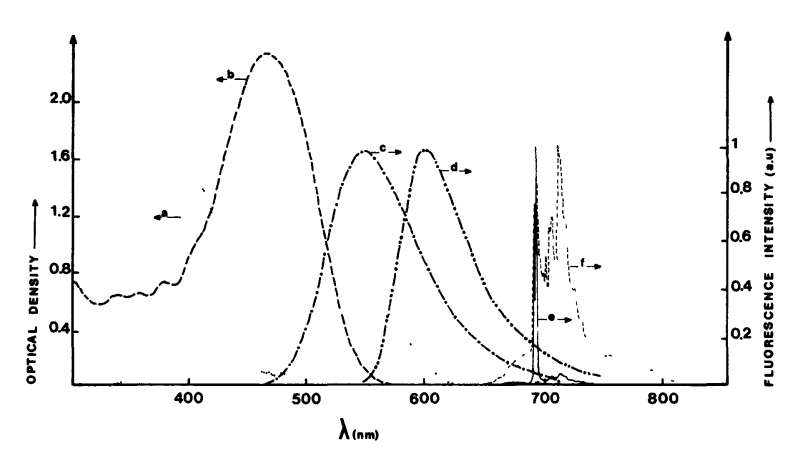

Fig. 5. - Absorption and emission spectra of ruby and DCM (in PMMA). a, absorption spectrum of the ruby plate used in device $1 ; b$, absorption spectrum of the PMMA-DCM plate used in device 2 ; $c$, emission spectrum of DCM in a PMMA plate at a concentration of $10^{-6} \mathrm{~mol} .1^{-1}\left(\lambda_{\text {exc }}=461 \mathrm{~nm}\right)$; $d$, emission spectrum at the output edge of the PMMA-DCM plate used in device 2 under illumination by the HMI lamp ; e, emission spectrum of a ruby plate at low chromium concentration $\left(\lambda_{\text {exc }}=461 \mathrm{~nm}\right)$; f, emission spectrum at the output edge of the ruby plate used in device 1 under illumination by the HMI lamp. All the emission spectra are corrected and normalized to the same height at the maximum. The band width of the emission monochromator is $0.5 \mathrm{~nm}$.

5.2 Available fluX AT the OUTPUT OF DeVices 1 AND 2. - Under illumination by the HMI simulator, the wavelength dependence of the flux at the output of devices 1 and 2 was measured by means of the ICOFMP system. The results are given in figure 6 . In the emission range of device 1 , the ratio of the available flux at the output edge, to the incident flux is equal to 16.2 ; for device 2 , this ratio is equal to 3.9.

5.3 ROLE OF REFLECTORS. - Figure 7 illustrates the role of the back diffuse reflector (BR). In principe, this reflector should only give rise to an increase in absorption of the incident flux, and to a concomitant increase in output flux. However the spectra exhibit weak but significant reabsorption effects. In the case of ruby, changes in $R$ bands are revealed (Fig. 7A.c) when dividing the spectrum recorded with BR (Fig. 7A.b) by the spectrum recorded without BR (Fig. 7A.a). An enhancement by a factor of $1.73 \pm 0.03$ is observed which is in good agreement with the expected value 


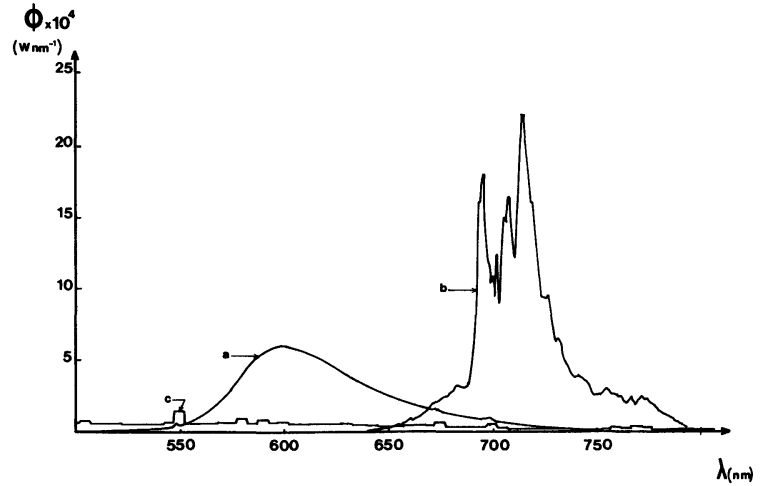

Fig. 6. - Available flux at the output edge of the ruby plate (b) and the PMMA-DCM plate (a) illuminated by the HMI lamp. Curve c represents the flux from the HMI lamp on an area identical with the output edge area of the plates over their emission range.
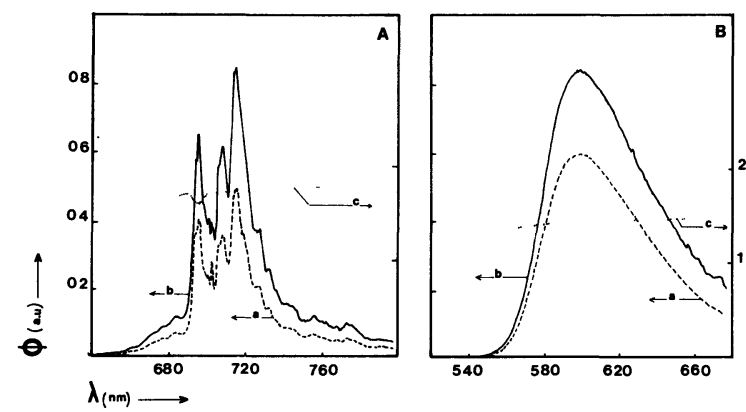

Fig. 7. - Available flux at the output edge of the ruby plate (A) and the PMMA-DCM plate (B) illuminated by the HMI lamp. Curves $a$ and $b$ are recorded without and with back reflector, respectively. Curves c represent the ratio b/a.

$(1.72 \pm 0.02)$ calculated from the ratio of the absorbed flux with and without BR.

In the case of PMMA doped with DCM (Fig. 7B.abc), the reabsorption effect is significant up to $590 \mathrm{~nm}$ and the enhancement factor due to the BR is equal to $1.41 \pm 0.04$, a value in good agreement with the theoretical value and with data given in the literature [22].

When decreasing the observation wavelength, a sharp increase in intensity is to be noted at the very beginning of the emission spectra (7A.c and 7B.c). In addition, a slight increase in intensity is observed at high wavelengths. These phenomena can be explained in terms of light reflected by the BR and transmitted by the plates towards the detector directly [22].

The efficiency of the opposite reflector (OR) that is coupled to the plate with a thin air gap, can be determined by using the ICOFMP system. The available flux at the two output edges of the plate are identical provided that the irradiance is homogeneous. Assuming that the reflection coefficient is equal to 1 (this assumption is valid in the present case [26]), the OR should cause the available flux to increase by a factor of 2. However this statement is correct if the Fresnel reflections are ignored and if only the high wavelength region of the spectrum is considered (where the reabsorption effect is negligible). Practically, the air gap has to be kept as thin as possible $(1 / 10 \mathrm{~mm}$ in the present case) because, for a perfect Lambertian reflector, the angular distribution of light intensity is a sphere tangent to the reflector. Thus, if the thickness of the air gap increases, a significant part of the scattered flux will not enter the FSC plate.

In the case of device 1 , the reabsorption effect is clearly shown for the $R$ band of ruby (Fig. 8a). At wavelengths higher than $710 \mathrm{~nm}$, the enhancement due to the OR is found to be constant and equal to $(50 \pm 3)$ $\%$ in terms of flux of photons.
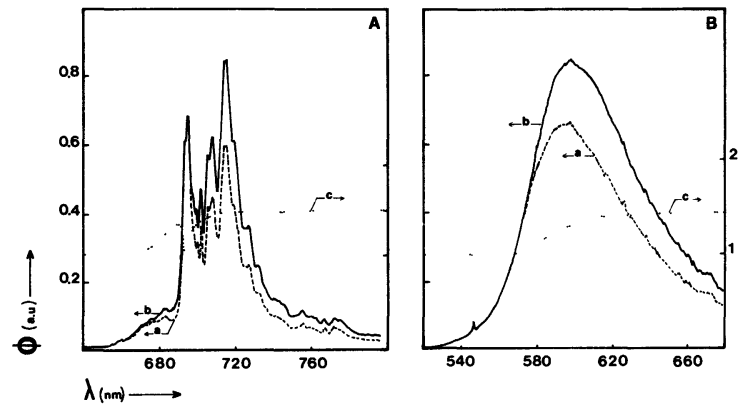

Fig. 8. - Available flux at the output edge of the ruby plate (A) and the PMMA-DCM plate (B) illuminated by the HMI lamp. Curves $a$ and $b$ are recorded without and with opposite reflector, respectively. Curves $c$ represent the ratio b/a.

As regards device 2, reabsorption is complete up to $565 \mathrm{~nm}$ (Fig 8B.c) i.e. up to the end of the absorption spectrum of DCM in PMMA (Fig. 4b). At wavelengths higher than $620 \mathrm{~nm}$, the enhancement of available flux is constant versus wavelength. This result is in good agreement with the Monte-Carlo simulation carried out by Sansregret and coworkers [15] which shows that reabsorption is negligible beyond $630 \mathrm{~nm}$. These observations confirm the validity of normalization in the high wavelength region of the emission spectra $[7,9]$. In device 2 , the enhancement due to the OR is $(40 \pm 3) \%$.

It should be noted that the positioning of the OR is critical in the determination of the gain, which explains large errors, as outlined above.

The results presented in figure 8 emphasize the role played by the reabsorption effect which is a serious limitation of FSC. Moreover, the expected enhancement by a factor of 2 due to the OR is not observed in the spectral region where there is no reabsorption. This fact can be explained in the case of PMMA plates by losses due to absorption of the matrix and to internal or surface defects (microscopic cracks, scratches, rough patches) which disturb the total internal reflection; some part of the trapped light is thus scattered out and lost. On the other hand, the optical quality of the ruby 
plate is excellent ; however a careful examination of the plate reveals significant variations in chromium concentration corresponding to growth layers. It is thus likely that some aggregates of chromium ions absorb photons without re-emission.

5.4 DETERMINATION OF FSC PARAMETERS. - In table I are reported the results concerning the concentration in number of photons $C_{n h \nu}$ (Eqs. (12) and (23)), the Stokes gain $G_{\mathrm{ST}}$ (Eqs. (13) and (24)), the gain in current intensity $\mathrm{G}_{\mathrm{I}}$ (Eqs. (16) and (25)) and the FSC quantum yield $\eta_{\text {FSC }}$ (Eq. (18)) for devices 1 and 2 .

The fraction $F_{\mathrm{a}}$ of the incident flux absorbed by the plate over the range of spectral sensitivity of the cell has been determined in the presence of the back reflector. The values are 0.424 and 0.403 for devices 1 and 2 , respectively, with a relative error of $2-3 \%$.

The values of the Stokes gain $G_{\mathrm{ST}}$ are 1.33 for the ruby plate and 1.27 for the DCM-PMMA plate. These results show that a red shift of the emission spectrum has only a slight effect on the FSC efficiency. Therefore, the importance of spectral matching to the cell sensitivity should not be emphasized. However, at constant flux of photons at the output edge of the FSC, heating of the cell is reduced when the emission range matches the range of spectral sensitivity of the cell, i.e. in the case of ruby. Consequently, when the doped plate together with the cell are employed as a second stage in solar concentrators using mirrors or Fresnel lenses, spectral matching allows to reduce the efficiency of the active or passive cooling system [20].

The concentration in number of photons $C_{n h \nu}$ is higher for the ruby plate (2.4) than for DCM-PMMA plate (1.5) at constant geometric gain $G$. The difference can be explained by the higher quantum yield of ruby which is close to 1 [24]. Drake et al. [9] have found a quantum yield of 0.81 for DCM in PMMA. Our measurements do not confirm this value : for an excitation wavelength of $480 \mathrm{~nm}$, the quantum yield of
DCM was found to be $0.39 \pm 0.02$ for a PMMA plate doped at low concentration $\left(10^{-6} \mathrm{~mol} .1^{-1}\right)$ as well as for the PMMA plate used in device 2. These measurements were carried out following the recommendations of Demas and Crosby [27] and using DCM in dimethylsulfoxide $(\eta=0.71[28])$ and quinine sulfate in 0.1 $\mathrm{NH}_{2} \mathrm{SO}_{4}(\eta=0.51$ [29] $)$ as standards. It is wortwhile noting that the quantum yield of DCM strongly depends on the excitation wavelength, in accordance with previous investigations on excitation spectra [5]. The discrepancy between our value and that of Drake et al., can be accounted for by differences in the polymerization process.

The determination of $C_{n h \nu}$ and $G_{\mathrm{ST}}$ allows us to calculate the expected values for the gain in current intensity $G_{\mathrm{I}}$ (Eq. (17)) which are 3.18 and 1.9 for devices 1 and 2 , respectively. It is of interest to compare these values with those obtained by direct determination, i.e. by comparing the short-circuit current of the edge-mounted cell (with an air gap of $1 / 10 \mathrm{~mm}$ ) and the short-circuit current measured with the same cell under direct insolation. The values of $G_{\text {Im }}$ obtained in this way (3.4 and 1.9 for devices 1 and 2 , respectively) are in good agreement with $G_{I}$ which demonstrates the validity and the thoroughness of our approach.

Finally, the quantum yield $\eta_{\text {FSC }}$ of devices 1 and 2 are $5.7 \%$ and $3.7 \%$, respectively, in the absence of fluid for optical matching. In the case of the PMMA plate doped with DCM, the value of $\eta_{\mathrm{FSC}}(3.7 \%)$ is to be compared with the value of $\eta_{\text {edge }}(3.5 \%)$ obtained by Drake et al. [9] with the same kind of plate, despite the fact that $\eta_{\text {FSC }}$ and $\eta_{\text {edge }}$ are not strictly equivalent (see Sect. 3) and that the experimental conditions are not the same.

The results obtained in the present work further confirm the outstanding performances of ruby. In a previous study [20], we have shown that the concentration factor in terms of power (i.e. the ratio of the power

Table I. - FSC characteristics.

\begin{tabular}{lcccccccc}
\hline & $n$ & $\theta_{\mathrm{c}}$ & $F_{\mathrm{a}}$ & $C_{n h v}$ & $G_{\text {ST }}$ & $G_{\text {I }}$ & $G_{\text {Im }}$ & $\eta_{\text {FSC }}$ \\
\hline Device 1 (ruby) & 1.76 & $34.6^{\circ}$ & 0.424 & 2.4 & 1.33 & 3.18 & 3.4 & $5.7 \%$ \\
Device 2 & 1.49 & $42.2^{\circ}$ & 0.403 & 1.5 & 1.27 & 1.9 & 1.9 & $3.7 \%$ \\
(DCM in PMMA) & & & & & & & & \\
\hline
\end{tabular}

\footnotetext{
$n \quad:$ index of refraction

$\theta_{c} \quad$ : critical angle for total internal reflection

$F_{\mathrm{a}} \quad$ : fraction of incident flux of photons absorbed by the plate

$C_{n h v}$ : concentration in number of photons

$G_{\mathrm{ST}}:$ Stokes gain

$G_{I}$ : gain in current intensity

$G_{\operatorname{Im}}$ : gain in current intensity (measured with the cell)

$\eta_{\text {FSC }}:$ quantum yield of the FSC
} 
delivered by the edge-mounted cell with an optical fluid to the power delivered by the same cell under direct insolation) is 15.4 .

\section{Conclusion.}

FSC performances have been evaluated with two types of plate made of either inorganic (ruby) or organic (DCM in PMMA) materials. A new photometric method using an integrating cylinder has been developed in order to obtain the absolute characteristics of the flux emitted by the plates.

The gain in current intensity delivered by the cell is the product of the concentration in number of photons and the Stokes gain. The results obtained with the two plates show that the Stokes gain, which characterizes the spectral matching to the cell sensitivity, is of minor importance whereas the major contribution to the gain is due to the number of available photons at the output edge of the plates.

A significant improvement in FSC efficiency is brought by the use of air-gap coupled diffuse reflectors. The experiments carried out with and without reflectors clearly reveal that the major limitations of FSC performances arise from the reabsorption effect and the defects of the matrix.

\section{Acknowledgments.}

The authors wish to thank Drs. J. Bastie and B. Rougié of the Institut National de Métrologie (CNAM) for their generous help in the calibration of the HMI lamp and for stimulating discussions. Thanks are also due to Dr. Ch. Perrin of CdF-Chimie for the manufacture of PMMA plates doped with DCM.

\section{Appendix.}

DETERMINATION OF THE FLUX ABSORBED BY A PLATE EQUIPPED WITH A BACK DIFFUSE REFLECTOR COUPLED WITH AN AIR GAP. - The back diffuse reflector is assumed to be ideal : the reflectivity $\rho$ is equal to 1 and the radiance $L\left(\mathrm{~W} \cdot \mathrm{m}^{-2} \cdot \mathrm{sr}^{-1}\right)$ is constant in all directions. The elementary flux coming from an area $S$ of this reflector in a solid angle within the cones of angle $\theta$ and $\theta+d \theta$ is given by :

$$
\mathrm{d} \phi=L S \cos \theta \mathrm{d} \Omega
$$

where $d \Omega=2 \pi \sin \theta d \theta$.

The total reflected flux $\phi(W)$ is equal to :

$$
\phi=\pi L S
$$

and the fraction of flux entering into the plate through its back face with an angle of incidence $\theta$, after the first reflection, is given by :

$$
\frac{\mathrm{d} \phi}{\phi}[1-r(\theta)]=[1-r(\theta)] \sin 2 \theta \mathrm{d} \theta
$$

where $r(\theta)$ is Fresnel's coefficient for natural light :

$$
r(\theta)=\frac{1}{2}\left[\frac{\sin ^{2}\left(\theta-\theta^{\prime}\right)}{\sin ^{2}\left(\theta+\theta^{\prime}\right)}+\frac{\tan ^{2}\left(\theta-\theta^{\prime}\right)}{\tan ^{2}\left(\theta+\theta^{\prime}\right)}\right]
$$

$\theta$ is the angle of incidence and $\theta^{\prime}$ the angle of refraction ( $\sin \theta=n \sin \theta^{\prime}$ ). It is assumed that the wavelength dependence of $r(\theta)$ can be ignored. The mean path length $e$ of photons inside the plate after reflection on the diffuse reflector is given by:

$$
\bar{e}=\frac{\int_{0}^{\pi / 2} \frac{e}{\cos \theta^{\prime}}[1-r(\theta)] \sin 2 \theta \mathrm{d} \theta}{\int_{0}^{\pi / 2}[1-r(\theta)] \sin 2 \theta \mathrm{d} \theta}
$$

where $e$ the thickness of the plate. In this calculation, the plate is assumed to be homogeneous and isotropic. Since the index of refraction is $n=1.49$ for PMMA and $n=1.76$ for ruby, we obtain $\bar{e}_{\mathrm{PMMA}}=1.14 e$ and $\bar{e}_{\text {ruby }}=1.09 e$.

As regards the transmittance of the plate, it can be written as follows

$$
T^{\prime}(\lambda)=\frac{\int_{0}^{\frac{\pi}{2}}[1-r(\theta)] \mathrm{e}^{-\frac{\alpha e}{\cos \theta}} \sin 2 \theta \mathrm{d} \theta}{\int_{0}^{\frac{\pi}{2}}[1-r(\theta)] \sin 2 \theta \mathrm{d} \theta} .
$$

Let $\phi_{\text {iss }}(\lambda)$ the incident spectral radiant flux normal to the upper face of the plate and $r$ the reflection coefficient normal to the plate ; the spectral flux transmitted through the back face of the plate [30] is given by :

$$
\phi_{1 \mathrm{~T}}(\lambda)=\phi_{\mathrm{iSS}}(\lambda) \frac{(1-r)^{2} T(\lambda)}{1-r^{2} T^{2}(\lambda)} .
$$

The term $r^{2} T^{2}(\lambda)$ can be neglected with respect to 1 ; hence

$$
\phi_{1 \mathrm{~T}}(\lambda) \simeq \phi_{\mathrm{iSS}}(\lambda) \cdot(1-r)^{2} T(\lambda) .
$$

It is easy to show that, taking into account multiple reflections and assuming that the transmitted beam is incoherent, the absorbed flux is given by :

$$
\phi_{1 \mathrm{a}}(\lambda)=\phi_{\mathrm{iss}}(\lambda) \frac{(1-r)[1-T(\lambda)]}{1-r T(\lambda)} .
$$

Additional absorption is due to the fact that the transmitted flux is reflected by the back diffuse reflector and re-enters into the plate. Moreover, multiple reflections of this type are to be considered, and for each scattered flux, the transmittance of the plate is $T^{\prime}(\lambda)$ as stated above. The additional absorbed flux $\phi_{2 \mathrm{a}}(\lambda)$ can be calculated as follows : 


$$
\begin{aligned}
\phi_{2 \mathrm{a}}(\lambda) & =\phi_{1 \mathrm{~T}}(\lambda)\left[1-T^{\prime}(\lambda)+r T^{\prime}(\lambda)-r T^{\prime 3}(\lambda)+r^{2} T^{\prime 3}(\lambda)-r^{2} T^{\prime 5}(\lambda)+r^{3} T^{\prime 5}(\lambda)-\cdots\right] \\
& =\phi_{1 \mathrm{~T}} \frac{1-T^{\prime}(\lambda)}{1-r T^{\prime}(\lambda)} .
\end{aligned}
$$

The total absorbed flux $\phi_{\mathrm{a}}$ is the sum $\phi_{1 \mathrm{a}}+\phi_{2 \mathrm{a}}$ :

$$
\begin{aligned}
\phi_{\mathrm{a}}(\lambda) & =\phi_{\mathrm{isS}} \frac{(1-r)[1-T(\lambda)]}{1-r T(\lambda)}+\phi_{\mathrm{iSS}}(1-r)^{2} T(\lambda)\left[\frac{1-T^{\prime}(\lambda)}{1-r T^{\prime}(\lambda)}\right] \\
& \simeq \phi_{\mathrm{iss}}(1-r)\left[1-\frac{T(\lambda) \cdot T^{\prime}(\lambda)}{1-r T^{\prime 2}(\lambda)}+\frac{2 r T(\lambda) \cdot T^{\prime}(\lambda)}{1-r T^{\prime 2}(\lambda)}-\frac{r T^{2}(\lambda)}{1-r T(\lambda)}\right] .
\end{aligned}
$$

Neglecting the terms in $r T^{2}$, we obtain

$$
\phi_{\mathrm{a}}(\lambda) \simeq \phi_{\mathrm{iss}}(1-r)\left[1-T(\lambda) \cdot T^{\prime}(\lambda)\right]
$$

and, over the wavelength range of spectral sensitivity of the cell, the total flux $\varphi_{\mathrm{a}}$ of photons absorbed by the plate is given by

$$
\varphi_{\mathrm{a}}=\int_{\mathrm{ss}} \phi_{\mathrm{iss}}(1-r)\left[1-T(\lambda) \cdot T^{\prime}(\lambda)\right] \frac{\lambda}{h c} \mathrm{~d} \lambda .
$$

\section{References}

[1] Weber, W. H., Lambe, J., Appl. Opt. 15 (1976) 2299.

[2] Levitt, J. A., Weber, W. H., Appl. Opt. 16 (1977) 2684.

[3] Goetzberger, A., Greubel, W., Appl. Phys. 14 (1977) 123.

[4] Batchelder, J. S., Zewail, A. H., Cole, T., Appl. Opt. 18 (1979) 3090.

[5] Batchelder, J. S., Zewail, A. H., Cole, T., Appl. Opt. 20 (1981) 3733.

[6] Filloux, A., Mugnier, J., Bourson, J., Valeur, B., Revue Phys. Appl. 18 (1983) 273.

[7] Roncali, J., Garnier, F., Appl. Opt. 23 (1984) 2809.

[8] Wood, J. R., Long, J. F., Conf. Record. of the 13th IEEE Photovolt. Conf. (1978) 1158.

[9] Drake, J. M., Lesiecki, M. L., SanSRegret, J., Thomas, W. R. L., Appl. Opt. 21 (1982) 2945.

[10] ReIsfeld, R., KAlisky, Y., Nature 283 (1980) 281.

[11] Reisfeld, R., NeumanN, S., Nature 274 (1978) 144.

[12] LeMPICKI, A., Appl. Opt. 22 (1983) 1160.

[13] Andrews, L. J., McCollum, B. C., LeMPicki, A., J. Lumin. 25 (1981) 877.

[14] Thomas, W. R. L., Drake, J. M., Lesiecki, M. L., Appl. Opt. 22 (1983) 3440.

[15] Sansregret, J., Drake, J. M., Thomas, W. R. L., LeSIECKI, M. L., Appl. Opt. 22 (1983) 573.

[16] Carrascosa, M., Unamuno, S., Agullo-Lopez, F., Appl. Opt. 22 (1983) 3236.
[17] Olson, R. W., Loring, R. F., FAyer, M. D., Appl. Opt. 20 (1981) 2934.

[18] Galluzzi, F., Scafé, E., Solar Energy 33 (1984) 501.

[19] Hovel, H. J., Hodgson, R. T., Woodall, J. M., Solar Energy Mat. 2 (1979) 19.

[20] Mugnier, J., Dordet, Y., Pouget, J., C.R. Heb. Séan. Acad. Sci. 301 (1985) 771.

[21] KeIL, G., Nucl. Instrum. Methods 87 (1970) 111.

[22] Roncali, J., Garnier, F., Solar Cells 13 (1984) 133.

[23] Goetzberger, A., Heidler, K., Wittwer, V., Zastrow, A., Baur, G., SaH, E., Proceedings of the second E.C. Photovolt. Solar Energy Conf. Berlin (1979) 515.

[24] Quimby, R. S., Yen, W. M., J. Appl. Phys. 51 (1980) 1780.

[25] Dodd, D. M., Wood, D. L., BARns, R. L., J. Appl. Phys. 35 (1964) 1183.

[26] Grum, F., Wightman, T. E., Appl. Opt. 16 (1977) 2775.

[27] Demas, J. N., Crosby, G. A., J. Phys. Chem. 75 (1971) 991.

[28] Hammond, P. R., Opt. Com. 29 (1979) 331.

[29] Velapoldi, R. A., Mielenz, K. D., N.B.S. special publication 260-64 (1984).

[30] Grum, F., Becherer, R. J., Optical Radiation Measurements. 1 Radiometry (Academic Press, New York) 1979. 